\title{
Convergence theorems on total asymptotically demicontractive and hemicontractive mappings in CAT(0) spaces
}

\author{
Xin-dong Liu' and Shih-sen Chang ${ }^{2 *}$ \\ Dedicated to Professor Shih-sen Chang on the occasion of his 80th birthday
}

${ }^{*}$ Correspondence:

changss2013@aliyun.com

${ }^{2}$ College of Statistics and

Mathematics, Yunnan University of

Finance and Economics, Kunming,

Yunnan 650221, China

Full list of author information is

available at the end of the article

\begin{abstract}
The purpose of this paper is to introduce the concepts of total asymptotically demicontractive mappings and total asymptotically hemicontractive mappings. Under suitable conditions some strong convergence theorems for these two kinds of mappings to converge to their fixed points in CAT(0) space are proved. The results presented in the paper extend and improve some recent results announced in the current literature.

MSC: $47 \mathrm{H} 09 ; 47 \mathrm{H} 10$

Keywords: total asymptotically demicontractive mapping; total asymptotically hemicontractive mappings; Ishikawa iterative scheme; Mann iterative scheme; CAT(0) space
\end{abstract}

\section{Introduction}

The fixed point theorems for nonexpansive mappings in the setting of CAT(0) space have been studied extensively by many authors (see, for example, Refs. [1-8]). Nanjaras and Panyanak [9], in 2010, obtained a $\triangle$-convergence theorem for asymptotically nonexpansive mappings in CAT(0) spaces. In 2012, Chang et al. [10] introduced the concept of total asymptotically nonexpansive mappings and proved the demiclosed principle for total asymptotically nonexpansive mappings in $\mathrm{CAT}(0)$ spaces and obtained a $\triangle$-convergence theorem for the Krasnoselskii-Mann iteration. Recently, Sahin and Basarir [11] obtained a strong convergence theorem for asymptotically quasi-nonexpansive mappings by a modified $S$-iteration.

The classes of asymptotically demicontractive mappings and asymptotically hemicontractive mappings were introduced in 1987 by Liu [12] in Hilbert spaces. Liu [13] obtained some convergence results of the Mann iterative scheme for the class of asymptotically demicontractive mappings. Osilike [14] in 1998 extended the results of Liu [13] to more general $q$-uniformly smooth Banach spaces. Zegeye et al. [15] in 2011 obtained some strong convergence results of the Ishikawa-type iterative scheme for the class of asymptotically pseudocontractive mappings in the intermediate sense without resorting to the hybrid method which was the main tool of Qin et al. [16]. Olaleru and Okeke [17] in 2012 established a strong convergence of Noor-type scheme for uniformly $L$-Lipschitzian and

○2014 Liu and Chang; licensee Springer. This is an Open Access article distributed under the terms of the Creative Commons Attribution License (http://creativecommons.org/licenses/by/2.0), which permits unrestricted use, distribution, and reproduction in any medium, provided the original work is properly cited. 
asymptotically pseudocontractive mappings in the intermediate sense without assuming any form of compactness.

Inspired and motivated by the recent work of Olaleru and Okeke [18], Chang et al. [10], Sahin and Basarir [11], the purpose of this paper is to introduce the concept of total asymptotically demicontractive mappings and total asymptotically hemicontractive mappings in CAT(0) spaces, and prove some strong convergence theorems of Mann- and Ishikawatype iterative schemes for uniformly $L$-Lipschitzian total asymptotically demicontractive mappings and total asymptotically hemicontractive mappings. The result presented in the paper extend and improve the corresponding results in Chang et al. [10], Sahin and Basarir [11], Liu [12, 13], Osilike [14] and Olaleru et al. [17, 18].

\section{Preliminaries and lemmas}

Let $(X, d)$ be a metric space. A geodesic path joining $x \in X$ to $y \in Y$ (or, more briefly, a geodesic from $x$ to $y$ ) is a map $c:[0, l] \rightarrow X$ such that $c(0)=x, c(l)=y$, and $d\left(c(t), c\left(t^{\prime}\right)\right)=$ $\left|t-t^{\prime}\right|$ for all $t, t^{\prime} \in[0, l]$. In particular, $c$ is an isometry and $d(x, y)=l$. The image $\alpha$ of $c$ is called a geodesic (or metric) segment joining $x$ and $y$. When it is unique, this geodesic segment is denoted by $[x, y]$. The space $(X, d)$ is said to be a geodesic space if every two points of $X$ are joined by a geodesic, and $X$ is said to be uniquely geodesic if there is exactly one geodesic joining $x$ and $y$ for each $x, y \in X$. A subset $Y \subset X$ is said to be convex if $Y$ includes every geodesic segment joining any two of its points.

Let $x, y \in X$, by [8, Lemma 2.1(iv)] for each $t \in[0,1]$, then there exists a unique point $z \in[x, y]$ such that

$$
d(x, z)=t d(x, y), \quad d(y, z)=(1-t) d(x, y) .
$$

From now on, we will use the notation $(1-t) x \oplus t y$ to denote the unique point $z$ satisfying (2.1).

The following lemma plays an important role in our paper.

Lemma 2.1 [8] A geodesic space $X$ is a CAT(0) space, if and only if the following inequality holds:

$$
d^{2}((1-t) x \oplus t y, z) \leq(1-t) d^{2}(x, z)+t d^{2}(y, z)-t(1-t) d^{2}(x, y)
$$

for all $x, y, z \in X$ and all $t \in[0,1]$. In particular, if $x, y, z$ are points in $a C A T(0)$ space and $t \in[0,1]$, then

$$
d((1-t) x \oplus t y, z) \leq(1-t) d(x, z)+t d(y, z) .
$$

Let $(X, d)$ be a metric space, $C$ be a nonempty subset of $X$. Recall a mapping $T: C \rightarrow C$ is said to be nonexpansive if

$$
d(T x, T y) \leq d(x, y), \quad \forall x, y \in C .
$$

$T$ is said to be asymptotically nonexpansive, if there is a sequence $\left\{k_{n}\right\} \subset[0, \infty)$ with $k_{n} \rightarrow 0$ such that

$$
d\left(T^{n} x, T^{n} y\right) \leq\left(1+k_{n}\right) d(x, y), \quad \forall n \geq 1, x, y \in C .
$$


$T$ is said to be $\left(\left\{\mu_{n}\right\},\left\{v_{n}\right\}, \phi\right)$-total asymptotically nonexpansive [10], if there exist nonnegative sequences $\left\{\mu_{n}\right\},\left\{v_{n}\right\}$ with $\mu_{n} \rightarrow 0, v_{n} \rightarrow 0$ and a strictly increasing continuous function $\phi:[0, \infty) \rightarrow[0, \infty)$ with $\phi(0)=0$ such that

$$
d\left(T^{n} x, T^{n} y\right) \leq d(x, y)+\mu_{n} \phi(d(x, y))+v_{n}, \quad \forall n \geq 1, x, y \in C .
$$

$T$ is said to be quasi-nonexpansive, if $F(T) \neq \emptyset$ and

$$
d(T x, p) \leq d(x, p), \quad \forall x \in C, p \in F(T) .
$$

$T$ is said to be uniformly L-Lipschitzian, if there exists a constant $L>0$ such that

$$
d\left(T^{n} x, T^{n} y\right) \leq L d(x, y), \quad \forall n \geq 1, x, y \in C .
$$

$T$ is said to be completely continuous, if the image of each bounded subset in $C$ is contained in a compact subset of $C$.

Berg and Nikolaev [19] introduced the concept of quasilinearization as follows:

Let us formally denote a pair $(a, b) \in X \times X$ by $\overrightarrow{a b}$ and call it a vector. Then a quasilinearization is defined as a map $\langle\cdot, \cdot\rangle:(X \times X) \times(X \times X) \rightarrow \mathbb{R}$ which is defined by

$$
\langle\overrightarrow{a b}, \overrightarrow{c d}\rangle=\frac{1}{2}\left(d^{2}(a, d)+d^{2}(b, c)-d^{2}(a, c)-d^{2}(b, d)\right), \quad \forall a, b, c, d \in X
$$

It is easily seen that $\langle\overrightarrow{a b}, \overrightarrow{c d}\rangle=\langle\overrightarrow{c d}, \overrightarrow{a b}\rangle,\langle\overrightarrow{a b}, \overrightarrow{c d}\rangle=-\langle\overrightarrow{b a}, \overrightarrow{c d}\rangle$, and $\langle\overrightarrow{a x}, \overrightarrow{c d}\rangle+\langle\overrightarrow{x b}, \overrightarrow{c d}\rangle=\langle\overrightarrow{a b}, \overrightarrow{c d}\rangle$ for all $a, b, c, d, x \in X$. We say that $X$ satisfies the Cauchy-Schwarz inequality if

$$
\langle\overrightarrow{a b}, \overrightarrow{c d}\rangle \leq d(a, b) d(c, d)
$$

for all $a, b, c, d \in X$. It is well known [19, Corollary 3$]$ that a geodesically connected metric space is a CAT(0) space if and only if it satisfies the Cauchy-Schwarz inequality.

By using the quasilinearization, we can define demicontractive mappings in CAT(0) spaces.

Definition 2.2 Let $X$ be a CAT(0) space, $C$ be a nonempty subset of $X$. A mapping $T$ : $C \rightarrow C$ is said to be demicontractive if $F(T) \neq \emptyset$ and there exists a constant $k \in(0,1]$ such that

$$
\langle\overrightarrow{T x p}, \overrightarrow{x p}\rangle \leq d^{2}(x, p)-k d^{2}(x, T x), \quad \forall x \in C, p \in F(T) .
$$

It is easy to show that (2.6) is equivalent to

$$
d^{2}(T x, p) \leq d^{2}(x, p)+(1-2 k) d^{2}(x, T x) .
$$

Remark 2.3 From the definitions, we may conclude that each quasi-expansive mapping is a demicontractive mapping with $k=\frac{1}{2}$.

Definition 2.4 Let $X$ be a CAT(0) space, $C$ be a nonempty subset of $X$. A mapping $T$ : $C \rightarrow C$ with $F(T) \neq \emptyset$ is said to be: 
(1) an asymptotically demicontractive mapping if there exist a constant $k \in[0,1)$ and a nonnegative sequence $\left\{\mu_{n}\right\} \subset[0, \infty)$ with $\mu_{n} \rightarrow 0$ such that

$$
d^{2}\left(T^{n} x, p\right) \leq\left(1+\mu_{n}\right) d^{2}(x, p)+k d^{2}\left(x, T^{n} x\right)
$$

for all $n \geq 1, x \in C, p \in F(T)$;

(2) an asymptotically demicontractive mapping in the intermediate sense if there exist a constant $k \in[0,1)$ and nonnegative sequences $\left\{\mu_{n}\right\},\left\{v_{n}\right\} \subset[0, \infty)$ with $\mu_{n} \rightarrow 0$, $v_{n} \rightarrow 0$ such that

$$
d^{2}\left(T^{n} x, p\right) \leq\left(1+\mu_{n}\right) d^{2}(x, p)+k d^{2}\left(x, T^{n} x\right)+v_{n}
$$

for all $n \geq 1, x \in C, p \in F(T)$;

(3) $a\left(\left\{\mu_{n}\right\},\left\{v_{n}\right\}, \phi\right)$-total asymptotically demicontractive mapping if there exist a constant $k \in[0,1)$ and nonnegative sequences $\left\{\mu_{n}\right\},\left\{v_{n}\right\} \subset[0, \infty)$ with $\mu_{n} \rightarrow 0$, $v_{n} \rightarrow 0$, and a strictly increasing continuous function $\phi:[0, \infty) \rightarrow[0, \infty)$ with $\phi(0)=0$ such that

$$
d^{2}\left(T^{n} x, p\right) \leq d^{2}(x, p)+\mu_{n} \phi(d(x, p))+k d^{2}\left(x, T^{n} x\right)+v_{n},
$$

for all $n \geq 1, x \in C, p \in F(T) ;$

(4) $a\left(\left\{\mu_{n}\right\},\left\{v_{n}\right\}, \phi\right)$-total asymptotically hemicontractive mapping if there exist nonnegative sequences $\left\{\mu_{n}\right\},\left\{v_{n}\right\} \subset[0, \infty)$ with $\mu_{n} \rightarrow 0, v_{n} \rightarrow 0$ and a strictly increasing continuous function $\phi:[0, \infty) \rightarrow[0, \infty)$ with $\phi(0)=0$ such that

$$
d^{2}\left(T^{n} x, p\right) \leq d^{2}(x, p)+\mu_{n} \phi(d(x, p))+d^{2}\left(x, T^{n} x\right)+v_{n}
$$

for all $n \geq 1, x \in C, p \in F(T)$.

Remark 2.5 From the definitions, it is easy to see that each asymptotically demicontractive mapping is an asymptotically demicontractive mapping in the intermediate sense with sequence $\left\{v_{n}=0\right\}$, and each asymptotically demicontractive mapping in the intermediate sense is a total asymptotically demicontractive mapping with $\phi(t)=t^{2}$.

Let $C$ be a nonempty bounded closed convex subset of a complete CAT $(0)$ space $X$ and $T: C \rightarrow C$ be a completely continuous and uniformly $L$-Lipschitzian and total asymptotically demicontractive or hemicontractive mapping with $F(T) \neq \emptyset$. We introduce the Mann-type iteration process,

$$
\begin{aligned}
& x_{1}=x \in C, \\
& x_{n+1}=\alpha_{n} T^{n} x_{n} \oplus\left(1-\alpha_{n}\right) x_{n}, \quad n \geq 1,
\end{aligned}
$$

and the Ishikawa-type iteration process,

$$
\begin{aligned}
& x_{1}=x \in C, \\
& y_{n}=\beta_{n} T^{n} x_{n} \oplus\left(1-\beta_{n}\right) x_{n}, \\
& x_{n+1}=\alpha_{n} T^{n} y_{n} \oplus\left(1-\alpha_{n}\right) x_{n}, \quad n \geq 1,
\end{aligned}
$$


where $\left\{\alpha_{n}\right\},\left\{\beta_{n}\right\}$ are the sequences in $[0,1]$. Under suitable conditions, we prove that sequences $\left\{x_{n}\right\}$ generated by (2.10) and (2.11) converges strongly to a fixed point of $T$. The results presented in the paper extend and improve some recent results announced in the current literature.

The following lemmas will be useful in this study.

Lemma 2.6 [13] Let $\left\{a_{n}\right\}_{n=1}^{\infty},\left\{b_{n}\right\}_{n=1}^{\infty}$ be sequences of nonnegative real numbers satisfying $a_{n+1} \leq a_{n}+b_{n}, a_{n} \geq 0, \forall n \geq 1, \sum_{n=1}^{\infty} b_{n}<\infty$ and we have a subsequence $\left\{a_{n_{k}}\right\}_{k=1}^{\infty}$, converging to 0 . Then we have

$$
\lim _{n \rightarrow \infty} a_{n}=0 .
$$

\section{Main results}

Theorem 3.1 Let $C$ be a nonempty bounded closed convex subset of a complete CAT(0) space $X$ and $T: C \rightarrow C$ be a completely continuous, uniformly L-Lipschitzian and $\left(\left\{\mu_{n}\right\},\left\{v_{n}\right\}, \phi\right)$-total asymptotically demicontractive mapping with $F(T) \neq \emptyset$. Let $\left\{x_{n}\right\}$ be the sequence defined by (2.10). If the following conditions are satisfied:

(i) $\sum_{n=1}^{\infty} \mu_{n}<\infty, \sum_{n=1}^{\infty} v_{n}<\infty$;

(ii) there exist positive constants $M$ and $M^{*}$, such that $\phi(t) \leq M^{*} t^{2}$ for all $t \geq M$;

(iii) $\epsilon \leq \alpha_{n} \leq 1-k-\epsilon, \forall n \geq 1$ for some $\epsilon>0$ and $k \in[0,1)$,

then $\left\{x_{n}\right\}$ converges strongly to a fixed point of $T$.

Proof Fix $p \in F(T)$, using (2.8), we obtain

$$
d^{2}\left(T^{n} x_{n}, p\right) \leq d^{2}\left(x_{n}, p\right)+\mu_{n} \phi\left(d\left(x_{n}, p\right)\right)+k d^{2}\left(x_{n}, T^{n} x_{n}\right)+v_{n} .
$$

Since $\phi$ is an increasing function, we have the result that $\phi(t) \leq \phi(M)$ if $t \leq M$ and $\phi(t) \leq$ $M^{*} t^{2}$ if $t \geq M$. In either case, we obtain

$$
\phi\left(d\left(x_{n}, p\right)\right) \leq \phi(M)+M^{*} d^{2}\left(x_{n}, p\right) .
$$

From (3.1), (3.2), and Lemma 2.1, we have

$$
\begin{aligned}
d^{2}\left(x_{n+1}, p\right)= & d^{2}\left(\alpha_{n} T^{n} x_{n} \oplus\left(1-\alpha_{n}\right) x_{n}, p\right) \\
\leq & \alpha_{n} d^{2}\left(T^{n} x_{n}, p\right)+\left(1-\alpha_{n}\right) d^{2}\left(x_{n}, p\right)-\alpha_{n}\left(1-\alpha_{n}\right) d^{2}\left(T^{n} x_{n}, x_{n}\right) \\
\leq & \alpha_{n}\left\{d^{2}\left(x_{n}, p\right)+\mu_{n} \phi\left(d\left(x_{n}, p\right)\right)+k d^{2}\left(x_{n}, T^{n} x_{n}\right)+v_{n}\right\} \\
& +\left(1-\alpha_{n}\right) d^{2}\left(x_{n}, p\right)-\alpha_{n}\left(1-\alpha_{n}\right) d^{2}\left(T^{n} x_{n}, x_{n}\right) \\
\leq & \alpha_{n}\left\{\left(1+\mu_{n} M^{*}\right) d^{2}\left(x_{n}, p\right)+k d^{2}\left(x_{n}, T^{n} x_{n}\right)+\mu_{n} \phi(M)+v_{n}\right\} \\
& +\left(1-\alpha_{n}\right) d^{2}\left(x_{n}, p\right)-\alpha_{n}\left(1-\alpha_{n}\right) d^{2}\left(T^{n} x_{n}, x_{n}\right) \\
= & \left(1+\alpha_{n} \mu_{n} M^{*}\right) d^{2}\left(x_{n}, p\right)+\alpha_{n} \mu_{n} \phi(M)+\alpha_{n} v_{n} \\
& -\alpha_{n}\left(1-k-\alpha_{n}\right) d^{2}\left(T^{n} x_{n}, x_{n}\right) .
\end{aligned}
$$


Now, we show that $\lim _{n \rightarrow \infty} d\left(T^{n} x_{n}, x_{n}\right)=0$. In fact, by condition (iii), we have $\alpha_{n} \geq \epsilon>0$, $1-k-\alpha_{n} \geq \epsilon$. Hence $\alpha_{n}\left(1-k-\alpha_{n}\right) \geq \epsilon^{2}>0$. It follows from (3.3) that

$$
\begin{aligned}
d^{2}\left(x_{n+1}, p\right) \leq & d^{2}\left(x_{n}, p\right)+\alpha_{n} \mu_{n} M^{*} d^{2}\left(x_{n}, p\right)+\alpha_{n} \mu_{n} \phi(M) \\
& -\epsilon^{2} d^{2}\left(T^{n} x_{n}, x_{n}\right)+\alpha_{n} v_{n} .
\end{aligned}
$$

Since $C$ is bounded, there exists a constant $K>0$ such that $d^{2}\left(x_{n}, p\right) \leq K, \forall n \geq 1$. It follows from (3.4) that

$$
d^{2}\left(x_{n+1}, p\right) \leq d^{2}\left(x_{n}, p\right)+\left(M^{*} K+\phi(M)\right) \mu_{n}-\epsilon^{2} d^{2}\left(T^{n} x_{n}, x_{n}\right)+v_{n}
$$

Hence,

$$
\epsilon^{2} d^{2}\left(T^{n} x_{n}, x_{n}\right) \leq d^{2}\left(x_{n}, p\right)-d^{2}\left(x_{n+1}, p\right)+\left(M^{*} K+\phi(M)\right) \mu_{n}+v_{n} .
$$

From (3.6), we have

$$
\begin{aligned}
\sum_{n=1}^{m} \epsilon^{2} d^{2}\left(T^{n} x_{n}, x_{n}\right) & \leq \sum_{n=1}^{m}\left[d^{2}\left(x_{n}, p\right)-d^{2}\left(x_{n+1}, p\right)+\left(M^{*} K+\phi(M)\right) \mu_{n}+v_{n}\right] \\
& =d^{2}\left(x_{1}, p\right)-d^{2}\left(x_{m+1}, p\right)+\left(M^{*} K+\phi(M)\right) \sum_{n=1}^{m} \mu_{n}+\sum_{n=1}^{m} v_{n} \\
& \leq 2 K+\left(M^{*} K+\phi(M)\right) \sum_{n=1}^{\infty} \mu_{n}+\sum_{n=1}^{\infty} v_{n} .
\end{aligned}
$$

Since $\left(M^{*} K+\phi(M)\right) \sum_{n=1}^{\infty} \mu_{n}+\sum_{n=1}^{\infty} v_{n}<\infty$, it follows that

$$
\lim _{n \rightarrow \infty} d\left(T^{n} x_{n}, x_{n}\right)=0
$$

Using (3.8), (2.10), and Lemma 2.1, we have

$$
d\left(x_{n+1}, x_{n}\right)=d\left(\alpha_{n} T^{n} x_{n} \oplus\left(1-\alpha_{n}\right) x_{n}, x_{n}\right)=\alpha_{n} d\left(T^{n} x_{n}, x_{n}\right) \rightarrow 0 \quad(n \rightarrow \infty) .
$$

Hence,

$$
\begin{aligned}
d\left(x_{n}, T x_{n}\right) & \leq d\left(x_{n}, x_{n+1}\right)+d\left(x_{n+1}, T^{n+1} x_{n+1}\right)+d\left(T^{n+1} x_{n+1}, T^{n+1} x_{n}\right)+d\left(T^{n+1} x_{n}, T x_{n}\right) \\
& \leq(1+L) d\left(x_{n+1}, x_{n}\right)+d\left(x_{n+1}, T^{n+1} x_{n+1}\right)+L d\left(T^{n} x_{n}, x_{n}\right) \\
& \rightarrow 0 \quad(n \rightarrow \infty) .
\end{aligned}
$$

Since $\left\{x_{n}\right\}_{n=1}^{\infty}$ is bounded and $T$ is completely continuous, there is a convergent subsequence $\left\{T x_{n_{r}}\right\}_{r=1}^{\infty}$ of $\left\{T x_{n}\right\}_{n=1}^{\infty}$ such that $T x_{n_{r}} \rightarrow q$ as $r \rightarrow \infty$. Since

$$
d\left(x_{n_{r}}, q\right) \leq d\left(x_{n_{r}}, T x_{n_{r}}\right)+d\left(T x_{n_{r}}, q\right) \rightarrow 0 \quad(r \rightarrow \infty)
$$

we have $x_{n_{r}} \rightarrow q$ as $r \rightarrow \infty$.

Since $T$ is continuous, we obtain $T q=q$, which shows that $q$ is a fixed point of $T$. The implies that $\left\{x_{n}\right\}_{n=1}^{\infty}$ has a subsequence which converges to a fixed point of $T$. 
In view of $\left(M^{*} K+\phi(M)\right) \sum_{n=1}^{\infty} \mu_{n}+\sum_{n=1}^{\infty} v_{n}<\infty$ and $\sum_{n=1}^{\infty} \epsilon^{2} d^{2}\left(T^{n} x_{n}, x_{n}\right)<\infty$, by Lemma 2.6, and (3.5), we have $\lim _{n \rightarrow \infty} d^{2}\left(x_{n}, q\right)=0$. Hence, $x_{n} \rightarrow q$ as $n \rightarrow \infty$. The proof of Theorem 3.1 is completed.

Theorem 3.2 Let $C$ be a nonempty bounded closed convex subset of a complete CAT(0) space $X$ and $T: C \rightarrow C$ be a completely continuous and uniformly L-Lipschitzian and $\left(\left\{\mu_{n}\right\},\left\{v_{n}\right\}, \phi\right)$-total asymptotically demicontractive mapping with $F(T) \neq \emptyset$. Let $\left\{x_{n}\right\}$ be a sequence defined by (2.11), where $\left\{\alpha_{n}\right\},\left\{\beta_{n}\right\} \in[0,1]$. Assume that the following conditions are satisfied:

(i) $\sum_{n=1}^{\infty} \mu_{n}<\infty, \sum_{n=1}^{\infty} v_{n}<\infty$;

(ii) there exist positive constants $M$ and $M^{*}$, such that $\phi(t) \leq M^{*} t^{2}$ for all $t \geq M$;

(iii) $\epsilon \leq k \leq \alpha_{n} \leq \beta_{n} \leq b, \forall n \geq 1$ for some $\epsilon>0, k \in[0,1)$ and some $b \in\left(0, L^{-2}\left[\sqrt{1+L^{2}}-1\right]\right)$.

Then $\left\{x_{n}\right\}$ converges strongly to a fixed point of $T$.

Proof Fixing $p \in F(T)$, using (2.8), (2.11), (3.2), and Lemma 2.1, we obtain

$$
\begin{aligned}
d^{2}\left(y_{n}, p\right)= & d^{2}\left(\beta_{n} T^{n} x_{n} \oplus\left(1-\beta_{n}\right) x_{n}, p\right) \\
\leq & \beta_{n} d^{2}\left(T^{n} x_{n}, p\right)+\left(1-\beta_{n}\right) d^{2}\left(x_{n}, p\right)-\beta_{n}\left(1-\beta_{n}\right) d^{2}\left(T^{n} x_{n}, x_{n}\right) \\
\leq & \beta_{n}\left\{d^{2}\left(x_{n}, p\right)+\mu_{n} \phi\left(d\left(x_{n}, p\right)\right)+k d^{2}\left(x_{n}, T^{n} x_{n}\right)+v_{n}\right\} \\
& +\left(1-\beta_{n}\right) d^{2}\left(x_{n}, p\right)-\beta_{n}\left(1-\beta_{n}\right) d^{2}\left(T^{n} x_{n}, x_{n}\right) \\
\leq & \beta_{n}\left\{\left(1+\mu_{n} M^{*}\right) d^{2}\left(x_{n}, p\right)+k d^{2}\left(x_{n}, T^{n} x_{n}\right)+\mu_{n} \phi(M)+v_{n}\right\} \\
+ & \left(1-\beta_{n}\right) d^{2}\left(x_{n}, p\right)-\beta_{n}\left(1-\beta_{n}\right) d^{2}\left(T^{n} x_{n}, x_{n}\right) \\
= & \left(1+\beta_{n} \mu_{n} M^{*}\right) d^{2}\left(x_{n}, p\right)+\beta_{n} \mu_{n} \phi(M)+\beta_{n} v_{n} \\
- & \beta_{n}\left(1-k-\beta_{n}\right) d^{2}\left(T^{n} x_{n}, x_{n}\right), \\
d^{2}\left(y_{n}, T^{n} y_{n}\right)= & d^{2}\left(\beta_{n} T^{n} x_{n} \oplus\left(1-\beta_{n}\right) x_{n}, T^{n} y_{n}\right) \\
\leq & \beta_{n} d^{2}\left(T^{n} x_{n}, T^{n} y_{n}\right)+\left(1-\beta_{n}\right) d^{2}\left(x_{n}, T^{n} y_{n}\right) \\
& -\beta_{n}\left(1-\beta_{n}\right) d^{2}\left(T^{n} x_{n}, x_{n}\right) \\
\leq & \beta_{n} L^{2} d^{2}\left(x_{n}, y_{n}\right)+\left(1-\beta_{n}\right) d^{2}\left(x_{n}, T^{n} y_{n}\right) \\
& -\beta_{n}\left(1-\beta_{n}\right) d^{2}\left(T^{n} x_{n}, x_{n}\right) \\
= & \beta_{n}^{3} L^{2} d^{2}\left(x_{n}, T^{n} x_{n}\right)+\left(1-\beta_{n}\right) d^{2}\left(x_{n}, T^{n} y_{n}\right) \\
& -\beta_{n}\left(1-\beta_{n}\right) d^{2}\left(T^{n} x_{n}, x_{n}\right) \\
= & \left(1-\beta_{n}\right) d^{2}\left(x_{n}, T^{n} y_{n}\right)-\beta_{n}\left(1-\beta_{n}-\beta_{n}^{2} L^{2}\right) d^{2}\left(T^{n} x_{n}, x_{n}\right) .
\end{aligned}
$$

Using (2.8), (3.2), (3.11), and (3.12), we obtain

$$
\begin{aligned}
d^{2}\left(T^{n} y_{n}, p\right) & \leq d^{2}\left(y_{n}, p\right)+\mu_{n} \phi\left(d\left(y_{n}, p\right)\right)+k d^{2}\left(y_{n}, T^{n} y_{n}\right)+v_{n} \\
& \leq d^{2}\left(y_{n}, p\right)+\mu_{n}\left[\phi(M)+M^{*} d^{2}\left(y_{n}, p\right)\right]+k d^{2}\left(y_{n}, T^{n} y_{n}\right)+v_{n} \\
& =\left(1+\mu_{n} M^{*}\right) d^{2}\left(y_{n}, p\right)+k d^{2}\left(y_{n}, T^{n} y_{n}\right)+\mu_{n} \phi(M)+v_{n}
\end{aligned}
$$




$$
\begin{aligned}
\leq & \left(1+\mu_{n} M^{*}\right)\left\{\left(1+\beta_{n} \mu_{n} M^{*}\right) d^{2}\left(x_{n}, p\right)+\beta_{n} \mu_{n} \phi(M)\right. \\
& \left.+\beta_{n} v_{n}-\beta_{n}\left(1-k-\beta_{n}\right) d^{2}\left(T^{n} x_{n}, x_{n}\right)\right\} \\
& +k\left\{\left(1-\beta_{n}\right) d^{2}\left(x_{n}, T^{n} y_{n}\right)-\beta_{n}\left(1-\beta_{n}-\beta_{n}^{2} L^{2}\right) d^{2}\left(T^{n} x_{n}, x_{n}\right)\right\} \\
& +\mu_{n} \phi(M)+v_{n} \\
= & \left(1+\mu_{n} M^{*}\right)\left(1+\beta_{n} \mu_{n} M^{*}\right) d^{2}\left(x_{n}, p\right)+\left(1+\mu_{n} M^{*}\right) \beta_{n} \mu_{n} \phi(M) \\
& +\left(1+\mu_{n} M^{*}\right) \beta_{n} v_{n}-\left(1+\mu_{n} M^{*}\right) \beta_{n}\left(1-k-\beta_{n}\right) d^{2}\left(T^{n} x_{n}, x_{n}\right) \\
& +k\left(1-\beta_{n}\right) d^{2}\left(x_{n}, T^{n} y_{n}\right)-k \beta_{n}\left(1-\beta_{n}-\beta_{n}^{2} L^{2}\right) d^{2}\left(T^{n} x_{n}, x_{n}\right) \\
& +\mu_{n} \phi(M)+v_{n} .
\end{aligned}
$$

Using (3.13), Lemma 2.1, and condition (iii), we obtain

$$
\begin{aligned}
& d^{2}\left(x_{n+1}, p\right)=d^{2}\left(\alpha_{n} T^{n} y_{n} \oplus\left(1-\alpha_{n}\right) x_{n}, p\right) \\
& \leq \alpha_{n} d^{2}\left(T^{n} y_{n}, p\right)+\left(1-\alpha_{n}\right) d^{2}\left(x_{n}, p\right)-\alpha_{n}\left(1-\alpha_{n}\right) d^{2}\left(T^{n} y_{n}, x_{n}\right) \\
& \leq \alpha_{n}\left\{\left(1+\mu_{n} M^{*}\right)\left(1+\beta_{n} \mu_{n} M^{*}\right) d^{2}\left(x_{n}, p\right)+\left(1+\mu_{n} M^{*}\right) \beta_{n} \mu_{n} \phi(M)\right. \\
& \times\left(1+\mu_{n} M^{*}\right) \beta_{n} v_{n}-\left(1+\mu_{n} M^{*}\right) \beta_{n}\left(1-k-\beta_{n}\right) d^{2}\left(T^{n} x_{n}, x_{n}\right) \\
& +k\left(1-\beta_{n}\right) d^{2}\left(x_{n}, T^{n} y_{n}\right)-k \beta_{n}\left(1-\beta_{n}-\beta_{n}^{2} L^{2}\right) d^{2}\left(T^{n} x_{n}, x_{n}\right) \\
& \left.+\mu_{n} \phi(M)+v_{n}\right\}+\left(1-\alpha_{n}\right) d^{2}\left(x_{n}, p\right)-\alpha_{n}\left(1-\alpha_{n}\right) d^{2}\left(T^{n} y_{n}, x_{n}\right) \\
& =\left[1+\alpha_{n} \mu_{n} M^{*}\left(1+\beta_{n}\left(1+\mu_{n} M^{*}\right)\right)\right] d^{2}\left(x_{n}, p\right) \\
& -\alpha_{n} \beta_{n}\left[k\left(1-\beta_{n}-\beta_{n}^{2} L^{2}\right)+\left(1+\mu_{n} M^{*}\right)\left(1-k-\beta_{n}\right)\right] d^{2}\left(T^{n} x_{n}, x_{n}\right) \\
& +\alpha_{n}\left[k\left(1-\beta_{n}\right)-\left(1-\alpha_{n}\right)\right] d^{2}\left(T^{n} y_{n}, x_{n}\right) \\
& +\alpha_{n}\left[1+\beta_{n}\left(1+\mu_{n} M^{*}\right)\right] \phi(M) \mu_{n}+\alpha_{n}\left[1+\beta_{n}\left(1+\mu_{n} M^{*}\right)\right] v_{n} \\
& \leq\left[1+\alpha_{n} \mu_{n} M^{*}\left(1+\beta_{n}\left(1+\mu_{n} M^{*}\right)\right)\right] d^{2}\left(x_{n}, p\right) \\
& -\alpha_{n} \beta_{n}\left[k\left(1-\beta_{n}-\beta_{n}^{2} L^{2}\right)+\left(1+\mu_{n} M^{*}\right)\left(1-k-\beta_{n}\right)\right] d^{2}\left(T^{n} x_{n}, x_{n}\right) \\
& +\left[k\left(1-\beta_{n}\right)-\alpha_{n}\left(1-\alpha_{n}\right)\right] d^{2}\left(T^{n} y_{n}, x_{n}\right) \\
& +\alpha_{n}\left[1+\beta_{n}\left(1+\mu_{n} M^{*}\right)\right] \phi(M) \mu_{n}+\alpha_{n}\left[1+\beta_{n}\left(1+\mu_{n} M^{*}\right)\right] v_{n} \\
& \leq\left[1+\alpha_{n} \mu_{n} M^{*}\left(1+\beta_{n}\left(1+\mu_{n} M^{*}\right)\right)\right] d^{2}\left(x_{n}, p\right) \\
& -\alpha_{n} \beta_{n}\left[k\left(1-\beta_{n}-\beta_{n}^{2} L^{2}\right)+\left(1+\mu_{n} M^{*}\right)\left(1-k-\beta_{n}\right)\right] d^{2}\left(T^{n} x_{n}, x_{n}\right) \\
& +\alpha_{n}\left[1+\beta_{n}\left(1+\mu_{n} M^{*}\right)\right] \phi(M) \mu_{n}+\alpha_{n}\left[1+\beta_{n}\left(1+\mu_{n} M^{*}\right)\right] v_{n} .
\end{aligned}
$$

Observe that by condition (iii), $k\left(1-\beta_{n}\right)-\alpha_{n}\left(1-\alpha_{n}\right) \leq 0$, so that the term $d^{2}\left(T^{n} y_{n}, x_{n}\right)$ can be dropped. Hence, we obtain (3.14).

Next, we show that $\lim _{n \rightarrow \infty} d\left(T^{n} x_{n}, x_{n}\right)=0$. From (3.14), we have

$$
\begin{aligned}
& d^{2}\left(x_{n+1}, p\right)-d^{2}\left(x_{n}, p\right) \\
& \leq \alpha_{n} \mu_{n} M^{*}\left(1+\beta_{n}\left(1+\mu_{n} M^{*}\right)\right) d^{2}\left(x_{n}, p\right) \\
& \quad-\alpha_{n} \beta_{n}\left[k\left(1-\beta_{n}-\beta_{n}^{2} L^{2}\right)+\left(1+\mu_{n} M^{*}\right)\left(1-k-\beta_{n}\right)\right] d^{2}\left(T^{n} x_{n}, x_{n}\right) \\
& \quad+\alpha_{n}\left[1+\beta_{n}\left(1+\mu_{n} M^{*}\right)\right] \phi(M) \mu_{n}+\alpha_{n}\left[1+\beta_{n}\left(1+\mu_{n} M^{*}\right)\right] v_{n} .
\end{aligned}
$$


Since $\mu_{n} \rightarrow 0,\left\{\mu_{n}\right\}_{n=1}^{\infty}$ is bounded. Observe that $C$ is bounded, $\alpha_{n}, \beta_{n} \in[0,1], \phi(M)$, and $M^{*}$ are constants. Now $\left\{\alpha_{n}\left[1+\beta_{n}\left(1+\mu_{n} M^{*}\right)\right]\right\}_{n=1}^{\infty},\left\{\alpha_{n}\left[1+\beta_{n}\left(1+\mu_{n} M^{*}\right)\right] \phi(M)\right\}_{n=1}^{\infty}$, and $\left\{\alpha_{n} M^{*}\left(1+\beta_{n}\left(1+\mu_{n} M^{*}\right)\right) d^{2}\left(x_{n}, p\right)\right\}_{n=1}^{\infty}$ are bounded. Hence, there exists a constant $K>0$ such that

$$
0 \leq \alpha_{n}\left[1+\beta_{n}\left(1+\mu_{n} M^{*}\right)\right]\left(1+\phi(M)+M^{*} d^{2}\left(x_{n}, p\right)\right) \leq K .
$$

Using (3.15) and (3.16), we obtain

$$
\begin{aligned}
d^{2}\left(x_{n+1}, p\right)-d^{2}\left(x_{n}, p\right) \leq & K\left(\mu_{n}+v_{n}\right)-\alpha_{n} \beta_{n}\left[k\left(1-\beta_{n}-\beta_{n}^{2} L^{2}\right)\right. \\
& \left.+\left(1+\mu_{n} M^{*}\right)\left(1-k-\beta_{n}\right)\right] d^{2}\left(T^{n} x_{n}, x_{n}\right) .
\end{aligned}
$$

By condition (iii), $b \in\left(0, L^{-2}\left[\sqrt{1+L^{2}}-1\right]\right)$, this shows that $1+b L^{2}<\sqrt{1+L^{2}}$. On squaring both sides, after simplifying we obtain $\frac{1-2 b-b^{2} L^{2}}{2}>0$. Since $1+\mu_{n} M^{*} \rightarrow 1$, there exists a natural number $N$ such that, for $n>N$,

$$
\begin{aligned}
k & \left(1-\beta_{n}-\beta_{n}^{2} L^{2}\right)+\left(1+\mu_{n} M^{*}\right)\left(1-k-\beta_{n}\right) \\
& \geq\left(1+\mu_{n} M^{*}\right)(1-k)-\left(1+\mu_{n} M^{*}\right) \beta_{n}-k \beta_{n}^{2} L^{2} \\
& \geq 1-b-\left(1+\mu_{n} M^{*}\right) b-b^{2} L^{2} \\
& >\frac{1-2 b-b^{2} L^{2}}{2}>0 .
\end{aligned}
$$

Assuming that $\lim _{n \rightarrow \infty} d\left(T^{n} x_{n}, x_{n}\right) \neq 0$, there exist $\epsilon_{0}>0$ and a subsequence $\left\{x_{n_{r}}\right\}_{r=1}^{\infty}$ of $\left\{x_{n}\right\}_{n=1}^{\infty}$ such that

$$
d^{2}\left(T^{n_{r}} x_{n_{r}}, x_{n_{r}}\right) \geq \epsilon_{0} .
$$

Without loss of generality, we can assume that $n_{1}>N$. From (3.17), we obtain

$$
\begin{aligned}
& \alpha_{n} \beta_{n}\left[k\left(1-\beta_{n}-\beta_{n}^{2} L^{2}\right)+\left(1+\mu_{n} M^{*}\right)\left(1-k-\beta_{n}\right)\right] d^{2}\left(T^{n} x_{n}, x_{n}\right) \\
& \quad \leq d^{2}\left(x_{n}, p\right)-d^{2}\left(x_{n+1}, p\right)+K\left(\mu_{n}+v_{n}\right) .
\end{aligned}
$$

Hence,

$$
\begin{aligned}
& \sum_{l=1}^{r} \alpha_{n_{l}} \beta_{n_{l}}\left[k\left(1-\beta_{n_{l}}-\beta_{n_{l}}^{2} L^{2}\right)+\left(1+\mu_{n_{l}} M^{*}\right)\left(1-k-\beta_{n_{l}}\right)\right] d^{2}\left(T^{n_{l}} x_{n_{l}}, x_{n_{l}}\right) \\
& \quad \leq \sum_{m=n_{1}}^{n_{r}} \alpha_{m} \beta_{m}\left[k\left(1-\beta_{m}-\beta_{m}^{2} L^{2}\right)+\left(1+\mu_{m} M^{*}\right)\left(1-k-\beta_{m}\right)\right] d^{2}\left(T^{m} x_{m}, x_{m}\right) \\
& \quad \leq \sum_{m=n_{1}}^{n_{r}}\left[d^{2}\left(x_{m}, p\right)-d^{2}\left(x_{m+1}, p\right)+K\left(\mu_{m}+v_{m}\right)\right] \\
& \quad=d^{2}\left(x_{n_{1}}, p\right)-d^{2}\left(x_{n_{r}+1}, p\right)+\sum_{m=n_{1}}^{n_{r}} K\left(\mu_{m}+v_{m}\right) .
\end{aligned}
$$


It follows from (3.18), (3.19), and (3.20) that

$$
r \epsilon^{2}\left(\frac{1-2 b-b^{2} L^{2}}{2}\right) \epsilon_{0} \leq d^{2}\left(x_{n_{1}}, p\right)-d^{2}\left(x_{n_{r}+1}, p\right)+\sum_{m=n_{1}}^{n_{r}} K\left(\mu_{m}+v_{m}\right) .
$$

Observing that $\sum_{n=1}^{\infty} K\left(\mu_{n}+v_{n}\right)<\infty$ and the boundedness of $C$, we see that the righthand side of (3.21) is bounded, the left-hand side of (3.21) is positively unbounded when $r \rightarrow \infty$. Hence, a contraction. Therefore

$$
\lim _{n \rightarrow \infty} d\left(T^{n} x_{n}, x_{n}\right)=0
$$

Using (2.1) and (2.11), we have

$$
\begin{aligned}
d\left(x_{n+1}, x_{n}\right)= & d\left(\alpha_{n} T^{n} y_{n} \oplus\left(1-\alpha_{n}\right) x_{n}, x_{n}\right) \\
& =\alpha_{n} d\left(T^{n} y_{n}, x_{n}\right) \\
& \leq d\left(T^{n} y_{n}, x_{n}\right) \\
& \leq d\left(T^{n} y_{n}, T^{n} x_{n}\right)+d\left(T^{n} x_{n}, x_{n}\right) \\
& \leq L d\left(y_{n}, x_{n}\right)+d\left(T^{n} x_{n}, x_{n}\right) \\
& =\beta_{n} L d\left(T^{n} x_{n}, x_{n}\right)+d\left(T^{n} x_{n}, x_{n}\right) \\
& \leq(1+L) d\left(T^{n} x_{n}, x_{n}\right) \\
& \rightarrow 0 \quad(n \rightarrow \infty) .
\end{aligned}
$$

Observe that

$$
\begin{aligned}
d\left(x_{n}, T x_{n}\right) \leq & d\left(x_{n}, x_{n+1}\right)+d\left(x_{n+1}, T^{n+1} x_{n+1}\right) \\
& +d\left(T^{n+1} x_{n+1}, T^{n+1} x_{n}\right)+d\left(T^{n+1} x_{n}, T x_{n}\right) \\
\leq & (1+L) d\left(x_{n+1}, x_{n}\right)+d\left(x_{n+1}, T^{n+1} x_{n+1}\right)+L d\left(T^{n} x_{n}, x_{n}\right) \\
\rightarrow & 0 \quad(n \rightarrow \infty) .
\end{aligned}
$$

Since $\left\{x_{n}\right\}_{n=1}^{\infty}$ is a bounded sequence and $T$ is completely continuous, there is a convergent subsequence $\left\{T x_{n_{r}}\right\}_{r=1}^{\infty}$ of $\left\{T x_{n}\right\}_{n=1}^{\infty}$. Let $T x_{n_{r}} \rightarrow q$ as $r \rightarrow \infty$. Then $x_{n_{r}} \rightarrow q$ as $r \rightarrow \infty$ since

$$
d\left(x_{n_{r}}, q\right) \leq d\left(x_{n_{r}}, T x_{n_{r}}\right)+d\left(T x_{n_{r}}, q\right) \rightarrow 0 \quad(r \rightarrow \infty) .
$$

From the continuity of $T$, we obtain $T q=q$, meaning that $q$ is a fixed point of $T$. Hence $\left\{x_{n}\right\}_{n=1}^{\infty}$ has a subsequence which converges to a fixed point of $T$.

Using (3.17) and (3.18), we see that there exists some natural number $N$ such that, for $n>N$,

$$
d^{2}\left(x_{n+1}, p\right) \leq d^{2}\left(x_{n}, p\right)+K\left(\mu_{n}+v_{n}\right)
$$

Noticing that $\sum_{n=1}^{\infty} K\left(\mu_{n}+v_{n}\right)<\infty$, it follows from Lemma 2.6 that $\lim _{n \rightarrow \infty} d^{2}\left(x_{n}, q\right)=0$. Hence, $x_{n} \rightarrow q$ as $n \rightarrow \infty$. The proof of Theorem 3.2 is completed. 
Theorem 3.3 Let $C$ be a nonempty bounded closed convex subset of a complete CAT(0) space $X$ and $T: C \rightarrow C$ be a completely continuous and uniformly L-Lipschitzian and $\left(\left\{\mu_{n}\right\},\left\{v_{n}\right\}, \phi\right)$-total asymptotically hemicontractive mapping with $F(T) \neq \emptyset$. Let $\left\{x_{n}\right\}$ be a sequence defined by (2.11), where $\left\{\alpha_{n}\right\},\left\{\beta_{n}\right\} \in[0,1]$. Assume that the following conditions are satisfied:

(i) $\sum_{n=1}^{\infty} \mu_{n}<\infty, \sum_{n=1}^{\infty} v_{n}<\infty$;

(ii) there exist positive constants $M$ and $M^{*}$, such that $\phi(t) \leq M^{*} t^{2}$ for all $t \geq M$;

(iii) $\epsilon \leq \alpha_{n} \leq \beta_{n} \leq b, \forall n \geq 1$ for some $\epsilon>0$, and some $b \in\left(0, L^{-2}\left[\sqrt{1+L^{2}}-1\right]\right)$.

Then $\left\{x_{n}\right\}$ converges strongly to a fixed point of $T$.

Proof Fix $p \in F(T)$, using (2.9), (2.11), (3.2), and Lemma 2.1, we obtain

$$
\begin{aligned}
d^{2}\left(y_{n}, p\right)= & d^{2}\left(\beta_{n} T^{n} x_{n} \oplus\left(1-\beta_{n}\right) x_{n}, p\right) \\
\leq & \beta_{n} d^{2}\left(T^{n} x_{n}, p\right)+\left(1-\beta_{n}\right) d^{2}\left(x_{n}, p\right)-\beta_{n}\left(1-\beta_{n}\right) d^{2}\left(T^{n} x_{n}, x_{n}\right) \\
\leq & \beta_{n}\left\{d^{2}\left(x_{n}, p\right)+\mu_{n} \phi\left(d\left(x_{n}, p\right)\right)+d^{2}\left(x_{n}, T^{n} x_{n}\right)+v_{n}\right\} \\
& +\left(1-\beta_{n}\right) d^{2}\left(x_{n}, p\right)-\beta_{n}\left(1-\beta_{n}\right) d^{2}\left(T^{n} x_{n}, x_{n}\right) \\
\leq & \beta_{n}\left\{\left(1+\mu_{n} M^{*}\right) d^{2}\left(x_{n}, p\right)+d^{2}\left(x_{n}, T^{n} x_{n}\right)+\mu_{n} \phi(M)+v_{n}\right\} \\
+ & \left(1-\beta_{n}\right) d^{2}\left(x_{n}, p\right)-\beta_{n}\left(1-\beta_{n}\right) d^{2}\left(T^{n} x_{n}, x_{n}\right) \\
= & \left(1+\beta_{n} \mu_{n} M^{*}\right) d^{2}\left(x_{n}, p\right)+\beta_{n} \mu_{n} \phi(M)+\beta_{n} v_{n} \\
+ & \beta_{n}^{2} d^{2}\left(T^{n} x_{n}, x_{n}\right), \\
d^{2}\left(y_{n}, T^{n} y_{n}\right)= & d^{2}\left(\beta_{n} T^{n} x_{n} \oplus\left(1-\beta_{n}\right) x_{n}, T^{n} y_{n}\right) \\
\leq & \beta_{n} d^{2}\left(T^{n} x_{n}, T^{n} y_{n}\right)+\left(1-\beta_{n}\right) d^{2}\left(x_{n}, T^{n} y_{n}\right) \\
& -\beta_{n}\left(1-\beta_{n}\right) d^{2}\left(T^{n} x_{n}, x_{n}\right) \\
\leq & \beta_{n} L^{2} d^{2}\left(x_{n}, y_{n}\right)+\left(1-\beta_{n}\right) d^{2}\left(x_{n}, T^{n} y_{n}\right) \\
& -\beta_{n}\left(1-\beta_{n}\right) d^{2}\left(T^{n} x_{n}, x_{n}\right) \\
= & \beta_{n}^{3} L^{2} d^{2}\left(x_{n}, T^{n} x_{n}\right)+\left(1-\beta_{n}\right) d^{2}\left(x_{n}, T^{n} y_{n}\right) \\
& -\beta_{n}\left(1-\beta_{n}\right) d^{2}\left(T^{n} x_{n}, x_{n}\right) \\
= & \left(1-\beta_{n}\right) d^{2}\left(x_{n}, T^{n} y_{n}\right)-\beta_{n}\left(1-\beta_{n}-\beta_{n}^{2} L^{2}\right) d^{2}\left(T^{n} x_{n}, x_{n}\right) .
\end{aligned}
$$

Using (2.9), (3.2), (3.25), and (3.26), we obtain

$$
\begin{aligned}
d^{2}\left(T^{n} y_{n}, p\right) \leq & d^{2}\left(y_{n}, p\right)+\mu_{n} \phi\left(d\left(y_{n}, p\right)\right)+d^{2}\left(y_{n}, T^{n} y_{n}\right)+v_{n} \\
\leq & \left(1+\mu_{n} M^{*}\right) d^{2}\left(y_{n}, p\right)+d^{2}\left(y_{n}, T^{n} y_{n}\right)+\mu_{n} \phi(M)+v_{n} \\
\leq & \left(1+\mu_{n} M^{*}\right)\left\{\left(1+\beta_{n} \mu_{n} M^{*}\right) d^{2}\left(x_{n}, p\right)+\beta_{n} \mu_{n} \phi(M)+\beta_{n} v_{n}\right. \\
& \left.+\beta_{n}^{2} d^{2}\left(T^{n} x_{n}, x_{n}\right)\right\}+\left(1-\beta_{n}\right) d^{2}\left(x_{n}, T^{n} y_{n}\right) \\
& -\beta_{n}\left(1-\beta_{n}-\beta_{n}^{2} L^{2}\right) d^{2}\left(T^{n} x_{n}, x_{n}\right)+\mu_{n} \phi(M)+v_{n} \\
= & \left(1+\mu_{n} M^{*}\right)\left(1+\beta_{n} \mu_{n} M^{*}\right) d^{2}\left(x_{n}, p\right)+\left(1+\mu_{n} M^{*}\right) \beta_{n} \mu_{n} \phi(M)
\end{aligned}
$$




$$
\begin{aligned}
& +\left(1+\mu_{n} M^{*}\right) \beta_{n} v_{n}+\left(1+\mu_{n} M^{*}\right) \beta_{n}^{2} d^{2}\left(T^{n} x_{n}, x_{n}\right) \\
& +\left(1-\beta_{n}\right) d^{2}\left(x_{n}, T^{n} y_{n}\right)-\beta_{n}\left(1-\beta_{n}-\beta_{n}^{2} L^{2}\right) d^{2}\left(T^{n} x_{n}, x_{n}\right) \\
& +\mu_{n} \phi(M)+v_{n} .
\end{aligned}
$$

Using (3.27), Lemma 2.1, and condition (iii), we obtain

$$
\begin{aligned}
d^{2}\left(x_{n+1}, p\right)= & d^{2}\left(\alpha_{n} T^{n} y_{n} \oplus\left(1-\alpha_{n}\right) x_{n}, p\right) \\
\leq & \alpha_{n} d^{2}\left(T^{n} y_{n}, p\right)+\left(1-\alpha_{n}\right) d^{2}\left(x_{n}, p\right)-\alpha_{n}\left(1-\alpha_{n}\right) d^{2}\left(T^{n} y_{n}, x_{n}\right) \\
\leq & \alpha_{n}\left\{\left(1+\mu_{n} M^{*}\right)\left(1+\beta_{n} \mu_{n} M^{*}\right) d^{2}\left(x_{n}, p\right)+\left(1+\mu_{n} M^{*}\right) \beta_{n} \mu_{n} \phi(M)\right. \\
& +\left(1+\mu_{n} M^{*}\right) \beta_{n} v_{n}+\left(1+\mu_{n} M^{*}\right) \beta_{n}^{2} d^{2}\left(T^{n} x_{n}, x_{n}\right) \\
& +\left(1-\beta_{n}\right) d^{2}\left(x_{n}, T^{n} y_{n}\right)-\beta_{n}\left(1-\beta_{n}-\beta_{n}^{2} L^{2}\right) d^{2}\left(T^{n} x_{n}, x_{n}\right) \\
& \left.+\mu_{n} \phi(M)+v_{n}\right\}+\left(1-\alpha_{n}\right) d^{2}\left(x_{n}, p\right)-\alpha_{n}\left(1-\alpha_{n}\right) d^{2}\left(T^{n} y_{n}, x_{n}\right) \\
= & {\left[1+\alpha_{n} \mu_{n} M^{*}\left(1+\beta_{n}\left(1+\mu_{n} M^{*}\right)\right)\right] d^{2}\left(x_{n}, p\right) } \\
& -\alpha_{n} \beta_{n}\left[\left(1-\beta_{n}-\beta_{n}^{2} L^{2}\right)-\beta_{n}\left(1+\mu_{n} M^{*}\right)\right] d^{2}\left(T^{n} x_{n}, x_{n}\right) \\
& +\alpha_{n}\left[\left(1-\beta_{n}\right)-\left(1-\alpha_{n}\right)\right] d^{2}\left(T^{n} y_{n}, x_{n}\right) \\
& +\alpha_{n}\left[1+\beta_{n}\left(1+\mu_{n} M^{*}\right)\right] \phi(M) \mu_{n}+\alpha_{n}\left[1+\beta_{n}\left(1+\mu_{n} M^{*}\right)\right] v_{n} \\
\leq & {\left[1+\alpha_{n} \mu_{n} M^{*}\left(1+\beta_{n}\left(1+\mu_{n} M^{*}\right)\right)\right] d^{2}\left(x_{n}, p\right) } \\
& -\alpha_{n} \beta_{n}\left[1-\beta_{n}-\beta_{n}^{2} L^{2}-\beta_{n}\left(1+\mu_{n} M^{*}\right)\right] d^{2}\left(T^{n} x_{n}, x_{n}\right) \\
& +\alpha_{n}\left[1+\beta_{n}\left(1+\mu_{n} M^{*}\right)\right] \phi(M) \mu_{n}+\alpha_{n}\left[1+\beta_{n}\left(1+\mu_{n} M^{*}\right)\right] v_{n} .
\end{aligned}
$$

Next, we show that $\lim _{n \rightarrow \infty} d\left(T^{n} x_{n}, x_{n}\right)=0$. From (3.28), we have

$$
\begin{aligned}
& d^{2}\left(x_{n+1}, p\right)-d^{2}\left(x_{n}, p\right) \\
& \leq \alpha_{n} \mu_{n} M^{*}\left(1+\beta_{n}\left(1+\mu_{n} M^{*}\right)\right) d^{2}\left(x_{n}, p\right) \\
& \quad-\alpha_{n} \beta_{n}\left[1-\beta_{n}-\beta_{n}^{2} L^{2}-\beta_{n}\left(1+\mu_{n} M^{*}\right)\right] d^{2}\left(T^{n} x_{n}, x_{n}\right) \\
& \quad+\alpha_{n}\left[1+\beta_{n}\left(1+\mu_{n} M^{*}\right)\right] \phi(M) \mu_{n}+\alpha_{n}\left[1+\beta_{n}\left(1+\mu_{n} M^{*}\right)\right] v_{n} .
\end{aligned}
$$

Since $\mu_{n} \rightarrow 0,\left\{\mu_{n}\right\}_{n=1}^{\infty}$ is bounded. Observe that $C$ is bounded, $\alpha_{n}, \beta_{n} \in[0,1], \phi(M)$ and $M^{*}$ are constants. Now $\left\{\alpha_{n}\left[1+\beta_{n}\left(1+\mu_{n} M^{*}\right)\right]\right\}_{n=1}^{\infty},\left\{\alpha_{n}\left[1+\beta_{n}\left(1+\mu_{n} M^{*}\right)\right] \phi(M)\right\}_{n=1}^{\infty}$, and $\left\{\alpha_{n} M^{*}\left(1+\beta_{n}\left(1+\mu_{n} M^{*}\right)\right) d^{2}\left(x_{n}, p\right)\right\}_{n=1}^{\infty}$ must be bounded. Hence, there exists a constant $K>0$ such that

$$
0 \leq \alpha_{n}\left[1+\beta_{n}\left(1+\mu_{n} M^{*}\right)\right]\left(1+\phi(M)+M^{*} d^{2}\left(x_{n}, p\right)\right) \leq K .
$$

Using (3.29) and (3.30), we obtain

$$
\begin{aligned}
d^{2}\left(x_{n+1}, p\right)-d^{2}\left(x_{n}, p\right) \leq & K\left(\mu_{n}+v_{n}\right) \\
& -\alpha_{n} \beta_{n}\left\{1-\beta_{n}-\beta_{n}^{2} L^{2}-\beta_{n}\left(1+\mu_{n} M^{*}\right)\right\} d^{2}\left(T^{n} x_{n}, x_{n}\right) .
\end{aligned}
$$


Observe that the condition $b \in\left(0, L^{-2}\left[\sqrt{1+L^{2}}-1\right]\right)$ implies that $b>0$ and $b<L^{-2} \times$ $\left[\sqrt{1+L^{2}}-1\right]$. This implies that $1+b L^{2}<\sqrt{1+L^{2}}$. On squaring both sides, we obtain $1+$ $2 b L^{2}+b^{2} L^{4}<1+L^{2}$, so we obtain $L^{2}-2 b L^{2}-b^{2} L^{4}>0$, and by dividing through by $L^{2}$, we obtain $1-2 b-b^{2} L^{2}>0$. Hence, $\frac{1-2 b-b^{2} L^{2}}{2}>0$. Since $1+\mu_{n} M^{*} \rightarrow 1$, there exists a natural number $N$ such that, for $n>N$,

$$
\begin{aligned}
1- & \beta_{n}-\beta_{n}^{2} L^{2}-\beta_{n}\left(1+\mu_{n} M^{*}\right) \\
& \geq 1-b-\left(1+\mu_{n} M^{*}\right) b-b^{2} L^{2} \\
& >\frac{1-2 b-b^{2} L^{2}}{2}>0 .
\end{aligned}
$$

Assuming that $\lim _{n \rightarrow \infty} d\left(T^{n} x_{n}, x_{n}\right) \neq 0$, then there exist $\epsilon_{0}>0$ and a subsequence $\left\{x_{n_{r}}\right\}_{r=1}^{\infty}$ of $\left\{x_{n}\right\}_{n=1}^{\infty}$ such that

$$
d^{2}\left(T^{n_{r}} x_{n_{r}}, x_{n_{r}}\right) \geq \epsilon_{0}
$$

Without loss of generality, we can assume that $n_{1}>N$. From (3.31), we obtain

$$
\begin{aligned}
& \alpha_{n} \beta_{n}\left[1-\beta_{n}-\beta_{n}^{2} L^{2}-\beta_{n}\left(1+\mu_{n} M^{*}\right)\right] d^{2}\left(T^{n} x_{n}, x_{n}\right) \\
& \quad \leq d^{2}\left(x_{n}, p\right)-d^{2}\left(x_{n+1}, p\right)+K\left(\mu_{n}+v_{n}\right) .
\end{aligned}
$$

Hence,

$$
\begin{aligned}
& \sum_{l=1}^{r} \alpha_{n_{l}} \beta_{n_{l}}\left[1-\beta_{n_{l}}-\beta_{n_{l}}^{2} L^{2}-\beta_{n_{l}}\left(1+\mu_{n_{l}} M^{*}\right)\right] d^{2}\left(T^{n_{l}} x_{n_{l}}, x_{n_{l}}\right) \\
& \quad \leq \sum_{m=n_{1}}^{n_{r}} \alpha_{m} \beta_{m}\left[1-\beta_{m}-\beta_{m}^{2} L^{2}-\beta_{m}\left(1+\mu_{m} M^{*}\right)\right] d^{2}\left(T^{m} x_{m}, x_{m}\right) \\
& \quad \leq \sum_{m=n_{1}}^{n_{r}}\left[d^{2}\left(x_{m}, p\right)-d^{2}\left(x_{m+1}, p\right)+K\left(\mu_{m}+v_{m}\right)\right] \\
& \quad=d^{2}\left(x_{n_{1}}, p\right)-d^{2}\left(x_{n_{r}+1}, p\right)+\sum_{m=n_{1}}^{n_{r}} K\left(\mu_{m}+v_{m}\right) .
\end{aligned}
$$

It follows from (3.32), (3.33), and (3.34) that

$$
\begin{aligned}
& r \epsilon^{2}\left(\frac{1-2 b-b^{2} L^{2}}{2}\right) \epsilon_{0} \\
& \quad \leq d^{2}\left(x_{n_{1}}, p\right)-d^{2}\left(x_{n_{r}+1}, p\right)+\sum_{m=n_{1}}^{n_{r}} K\left(\mu_{m}+v_{m}\right) .
\end{aligned}
$$

Observing that $\sum_{n=1}^{\infty} K\left(\mu_{n}+v_{n}\right)<\infty$ and the boundedness of $C$, we see that the righthand side of (3.35) is bounded, the left-hand side of (3.35) is positively unbounded when $r \rightarrow \infty$. Hence, a contraction. Therefore

$$
\lim _{n \rightarrow \infty} d\left(T^{n} x_{n}, x_{n}\right)=0
$$


Using (2.1) and (2.11), we have

$$
\begin{aligned}
d\left(x_{n+1}, x_{n}\right) & =d\left(\alpha_{n} T^{n} y_{n} \oplus\left(1-\alpha_{n}\right) x_{n}, x_{n}\right) \\
& =\alpha_{n} d\left(T^{n} y_{n}, x_{n}\right) \\
& \leq d\left(T^{n} y_{n}, x_{n}\right) \\
& \leq d\left(T^{n} y_{n}, T^{n} x_{n}\right)+d\left(T^{n} x_{n}, x_{n}\right) \\
& \leq L d\left(y_{n}, x_{n}\right)+d\left(T^{n} x_{n}, x_{n}\right) \\
& =\beta_{n} L d\left(T^{n} x_{n}, x_{n}\right)+d\left(T^{n} x_{n}, x_{n}\right) \\
& \leq(1+L) d\left(T^{n} x_{n}, x_{n}\right) \\
& \rightarrow 0 \quad(n \rightarrow \infty) .
\end{aligned}
$$

Hence,

$$
\begin{aligned}
d\left(x_{n}, T x_{n}\right) \leq & d\left(x_{n}, x_{n+1}\right)+d\left(x_{n+1}, T^{n+1} x_{n+1}\right) \\
& +d\left(T^{n+1} x_{n+1}, T^{n+1} x_{n}\right)+d\left(T^{n+1} x_{n}, T x_{n}\right) \\
\leq & (1+L) d\left(x_{n+1}, x_{n}\right)+d\left(x_{n+1}, T^{n+1} x_{n+1}\right)+L d\left(T^{n} x_{n}, x_{n}\right) \\
\rightarrow & 0 \quad(n \rightarrow \infty) .
\end{aligned}
$$

Since $\left\{x_{n}\right\}_{n=1}^{\infty}$ is a bounded sequence and $T$ is completely continuous, there is a convergent subsequence $\left\{T x_{n_{r}}\right\}_{r=1}^{\infty}$ of $\left\{T x_{n}\right\}_{n=1}^{\infty}$. Let $T x_{n_{r}} \rightarrow q$ as $r \rightarrow \infty$. Then $x_{n_{r}} \rightarrow q$ as $r \rightarrow \infty$ since

$$
d\left(x_{n_{r}}, q\right) \leq d\left(x_{n_{r}}, T x_{n_{r}}\right)+d\left(T x_{n_{r}}, q\right) \rightarrow 0 \quad(r \rightarrow \infty) .
$$

From the continuity of $T$, we obtain $T q=q$, meaning that $q$ is a fixed point of $T$. Hence $\left\{x_{n}\right\}_{n=1}^{\infty}$ has a subsequence which converges to a fixed point of $T$.

Using (3.31) and (3.32), we see that there exists some natural number $N$ such that, for $n>N$,

$$
d^{2}\left(x_{n+1}, p\right) \leq d^{2}\left(x_{n}, p\right)+K\left(\mu_{n}+v_{n}\right)
$$

Notice that $\sum_{n=1}^{\infty} K\left(\mu_{n}+v_{n}\right)<\infty$, it follows from Lemma 2.6 that

$$
\lim _{n \rightarrow \infty} d^{2}\left(x_{n}, q\right)=0
$$

Hence, $x_{n} \rightarrow q$ as $n \rightarrow \infty$. The proof of Theorem 3.3 is completed. 


\section{Author details}

'Institute of Mathematics, Yibin University, Yibin, Sichuan 644000, China. ${ }^{2}$ College of Statistics and Mathematics, Yunnan University of Finance and Economics, Kunming, Yunnan 650221, China.

\section{Acknowledgements}

The authors would like to express their thanks to the editors and the referees for their helpful comments and suggestions. This work is supported by the Scientific Research Fund of Science Technology Department of Sichuan Province (2011 JYZ010) and the Scientific Research Fund of Sichuan Provincial Education Department (13ZA0199) and the Foundation of National Natural Science Foundation of China (Grant No. 11361070).

\section{Received: 17 March 2014 Accepted: 20 October 2014 Published: 31 Oct 2014}

\section{References}

1. Kirk, WA: Geodesic geometry and fixed point theory. In: Seminar of Mathematical Analysis (Malaga/Seville, 2002-2003). Colec. Abierta, vol. 64, pp. 195-225. Univ. Sevilla Secr. Publ., Seville (2003)

2. Kirk, WA: Geodesic geometry and fixed point theory. II. In: International Conference on Fixed Point Theory and Applications, pp. 113-142. Yokohama Publ., Yokohama (2004)

3. Abkar, A, Eslamian, M: Common fixed point results in CAT(0) spaces. Nonlinear Anal. 74, 1835-1840 (2011)

4. Shahzad, N: Invariant approximations in CAT(0) spaces. Nonlinear Anal. 70, 4338-4340 (2009)

5. Dhompongsa, S, Kaewkhao, A, Panyanak, B: Lim's theorem for multivalued mappings in CAT(0) spaces. J. Math. Anal. Appl. 312, 478-487 (2005)

6. Shahzad, N, Markin, J: Invariant approximation for commuting mappings in hyperconvex and CAT(0) spaces. J. Math. Anal. Appl. 337, 1457-1464 (2008)

7. Shahzad, N: Fixed point results for multimaps in CAT(0) spaces. Topol. Appl. 156, 997-1001 (2009)

8. Dhompongsa, S, Panyanak, B: On D-convergence theorems in CAT(0) spaces. Comput. Math. Appl. 56(10), 2572-2579 (2008)

9. Nanjaras, B, Panyanak, B: Demiclosed principle for asymptotically nonexpansive mappings in CAT(0) spaces. Fixed Point Theory Appl. 2010, Article ID 268780 (2010)

10. Chang, SS, Wang, L, Joseph Lee, HW, Chan, CK, Yang, L: Total asymptotically nonexpansive mappings in CAT(0) space demiclosed principle and $\triangle$-convergence theorems for total asymptotically nonexpansive mappings in CAT(0) spaces. Appl. Math. Comput. 219, 2611-2617 (2012)

11. Sahin, A, Basarir, M: On the strong convergence of a S-iteration process for asymptotically quasi-nonexpansive mapping in a CAT(0) space. Fixed Point Theory Appl. 2013, Article ID 12 (2013). doi:10.1186/1687-1812-2013-12

12. Liu, QH: On Naimpally and Singh's open questions. J. Math. Anal. Appl. 124, 157-164 (1987)

13. Liu, QH: Convergence theorems of the sequence of iterates for asymptotically demicontractive and hemicontractive mappings. Nonlinear Anal., Theory Methods Appl. 26(11), 1835-1842 (1996)

14. Osilike, MO: Iterative approximation of fixed points of asymptotically demicontractive mappings. Indian J. Pure Appl. Math. 24, 1291-1300 (1998)

15. Zegeye, $\mathrm{H}$, Robdera, $\mathrm{M}$, Choudhary, $\mathrm{B}$ : Convergence theorems for asymptotically pseudocontractive mappings in the intermediate sense. Comput. Math. Appl. 62, 326-332 (2011)

16. Qin, X, Cho, SY, Kim, JK: Convergence theorems on asymptotically pseudocontractive mappings in the intermediate sense. Fixed Point Theory Appl. 2010, Article ID 186874 (2010). doi:10.1155/2010/186874

17. Olaleru, JO, Okeke, GA: Strong convergence theorems for asymptotically pseudocontractive mappings in the intermediate sense. Br. J. Math. Comput. Sci. 3(2), 151-162 (2012)

18. Olaleru, JO, Okeke, GA: Convergence theorems on asymptotically demicontractive and hemicontractive mappings in the intermediate sense. Fixed Point Theory Appl. 2013, Article ID 352 (2013)

19. Berg, ID, Nikolaev, IG: Quasilinearization and curvature of Alexandrov spaces. Geom. Dedic. 133, 195-218 (2008)

10.1186/1029-242X-2014-436

Cite this article as: Liu and Chang: Convergence theorems on total asymptotically demicontractive and hemicontractive mappings in CAT(0) spaces. Journal of Inequalities and Applications 2014, 2014:436

\section{Submit your manuscript to a SpringerOpen ${ }^{\mathcal{O}}$ journal and benefit from:}

- Convenient online submission

Rigorous peer review

- Immediate publication on acceptance

- Open access: articles freely available online

- High visibility within the field

- Retaining the copyright to your article 